\title{
Elastic and Thermal Dynamics in Femtosecond Laser-Induced Structural Change Inside Glasses Studied by the Transient Lens Method
}

\author{
Masaaki Sakakura, ${ }^{1}$ Masahide Terazima, ${ }^{2}$ Yasuhiko Shimotsuma, ${ }^{1}$ \\ Kiyotaka Miura, ${ }^{3}$ and Kazuyuki Hirao ${ }^{3}$ \\ ${ }^{1}$ Kyoto University Office of Society-Academia Collaboration for Innovation, Kyoto 615-8520, Japan \\ ${ }^{2}$ Department of Chemistry, Graduate School of Science, Kyoto University, Kyoto 606-8502, Japan \\ ${ }^{3}$ Department of Material Chemistry, Graduate School of Engineering, Kyoto University, Kyoto 615-8510, Japan
}

Correspondence should be addressed to Masahide Terazima, mterazima@kuchem.kyoto-u.ac.jp

Received 13 September 2010; Accepted 25 November 2010

Academic Editor: Chunlei Guo

Copyright ( 2010 Masaaki Sakakura et al. This is an open access article distributed under the Creative Commons Attribution License, which permits unrestricted use, distribution, and reproduction in any medium, provided the original work is properly cited.

\begin{abstract}
A technique to study femtosecond laser induced structural change inside glasses, the transient lens (TrL) method, is described. Because the TrL method is sensitive to the refractive index change around the photoexcited region, the time dependence of the density, pressure, and temperature changes, which accompany refractive index change, can be monitored over a broad range of timescales. In the picosecond-nanosecond time range, the pressure wave generation was observed as an oscillating $\operatorname{TrL}$ signal. By comparing the TrL signal with that calculated based on thermoelastic simulation, the density, pressure, and temperature changes in the photoexcited region can be estimated. In the longer time range (nanoseconds-milliseconds), the thermal diffusion process was observed. By fitting the TrL signal with that simulated based on thermal diffusion, the temporal evolution of the temperature distribution was obtained. Based on these observations, the features of femtosecond laser-induced structural change inside glasses are revealed. The advantages of the TrL method are described.
\end{abstract}

\section{Introduction}

Local structural change induced inside transparent materials using tightly focused femtosecond (fs) laser pulses enables us to fabricate various kinds of three-dimensional structures inside glasses, polymers, and inorganic crystals; this microfabrication technique with an fs laser has been used to produce small optical devices such as optical waveguides, photonic crystals, and 3D optical memory [1-7]. When an fs laser pulse is tightly focused inside a transparent material, only the material in the focal volume is photoexcited through nonlinear interaction with a strong laser field, resulting in localized permanent structural change inside the bulk of the material. It is widely known that when a strong laser pulse is focused in a material, the material exposed to the laser field is ejected and a less dense or void structure appears [8-11]. Such a structure is considered to be the result of an explosive expansion after the formation of high energy plasma and the subsequent energy transfer from hot electrons to the lattice. On the other hand, a structure with high refractive index has been observed around the laser focal region, when the photoexcitation occurs inside the material and the excitation laser energy is not high $[1,4-7,12]$. To control these structural changes and develop this fabrication technique, it is important to understand the mechanism of the structural change.

Several researchers have suggested that the fs laser focal region in a glass attains high temperature and high pressure immediately after irradiation based on observations of Raman scattering [12] and fluorescence [13] from the light-illuminated region. Chan et al. observed an increase in the Raman band intensity from the structure inside a silica glass created by a tightly focused femtosecond laser. They suggested that the irradiated region experiences high 
temperature after laser irradiation, because the Raman band intensity becomes larger as the fictive temperature of the glass becomes higher [12]. Watanabe et al. suggest that the temperature of the laser-irradiated region in a glass becomes higher than $1000 \mathrm{~K}$ based on an observation of a decrease in luminescence from the defects that are annihilated at high temperature [13]. Schaffer et al. also proposed that the morphology of the densified structure can be explained by a thermal effect [6]. Recently, Zhigilei et al. reported MD simulation of laser ablation and showed that temperature elevation immediately after the irradiation by a short laser pulse causes high pressure in the irradiated region and oscillations between compressive and expansive pressure occur $[14,15]$. The acoustic wave monitored by Kudryashov et al. also suggests that the photoexcited region is under high pressure after focusing fs laser pulse inside a glass [16].

These studies suggest that the pressure and temperature changes should play an important role in the fs laserinduced structural change inside glasses. Therefore, the timeresolved study of the pressure (or density) and temperature dynamics around the laser focal region should be essential for understanding the mechanism. In most time-resolved studies, light absorption, emission, or scattering is detected as a function of time. With these detection methods, it is difficult to extract information about pressure and temperature changes from these signals, because they are not sensitive to density, temperature, and pressure changes. Powerful techniques to detect density and temperature are transient lens (TrL) and transient grating (TG) methods [17-27]. In these methods, the refractive index changes induced by density and temperature changes are very sensitively detected using the diffraction of a probe light. So far, these methods have been used to measure the molar volume changes and released thermal energies in various chemical reactions [19, 26]. In this paper, we review investigations of the fs laserinduced structural change inside glasses by the TrL method. To observe the dynamics of this photoinduced process, the TrL method is suitable, because a refractive index lens is formed by one excitation beam whereas the TG method requires two beams to create the refractive index grating.

First, we explain the principle of the TrL method for observation of the dynamics of laser-induced structural change inside glasses. Because the refractive index lens in this study is not a simple lens-shape, the conventional analysis of the TrL method cannot be used. An alternative method for calculation of the TrL signal based on diffraction theory is presented. In the following section, the results from the TrL method in the picosecond time range will be shown. In this time range, the relaxation process of the thermal stress in the photoexcited region was observed. We found that the relaxation of the thermal stress resulted in the generation of a pressure wave. The observed pressure wave enables us to estimate the pressure change in the photoexcited region. In addition, the observation of the pressure wave by timeresolved light transmission imaging, which is a conventional method to detect the dynamics of laser processing, will be shown for comparison with the TrL method. Next, the observation of the thermal diffusion process in the longer time region (nanoseconds-milliseconds) will be described.
From these observations, the temporal evolution of the temperature distribution can be obtained and the effect of fast cooling can be evaluated based on the thermal diffusion. Finally, we summarize the dynamics that have been elucidated by the TrL method.

\section{Method}

2.1. Principle of the TrL method. The TrL method is one of the techniques for observing the dynamics of processes which are accompanied by a refractive index change (including change of chemical species, temperature change, and density change) [17-25]. A schematic drawing of the TrL method is shown in Figure 1(a). When the pump beam is passed through the sample, a refractive index lens appears due to the refractive index change which comes from the heat generation, density change, and chemical reaction. This refractive index lens is called a "TrL". To detect the TrL, a probe beam is introduced into the photoexcited region, and the transmission of the probe beam through an aperture is detected. In the situation shown in Figure $1(\mathrm{a})(d<0$, cf. Section 2.3), the transmission is larger than that before photoexcitation, because the probe beam is contracted at the aperture by the concave TrL. The temporal evolution of the transmission of the probe beam through an aperture is called a "TrL signal". Because the TrL includes changes in refractive index arising from various dynamic processes, the $\operatorname{TrL}$ signal provides us with information on the thermal energy or chemical species in a laser-induced reaction. On the other hand, the spatial profile of the probe beam is referred to as a "TrL image" and will be described in more detail later.

When a sample is photoexcited by a weak pump light and no elastic relaxation affects the refractive index lens, the spatial distribution of the refractive index change is the same as the spatial intensity distribution of the pump beam. When the spatial intensity distribution of the pump beam is Gaussian shaped and the lens effect is sufficiently small, the light intensity at the center of the probe beam is proportional to the refractive index change. In this case, a traditional analytical method for a thermal lens experiment can be used and the analysis of the TrL signal is rather simple. However, in the case of photoexcitation inside a glass by an fs laser pulse, the induced refractive index distribution will be complicated, because of the generation of a large amount of thermal energy and strong elastic waves. Therefore, we need to calculate the intensity distribution of the probe beam $(\operatorname{TrL}$ image) for an arbitrary refractive index distribution.

\subsection{TrL Method for fs Laser-Induced Dynamics Inside a Glass.} Figure 1(b) shows a schematic picture of the TrL detection for observation of the fs laser-induced dynamics inside a glass. When an fs laser pulse (pump pulse) is focused tightly inside a glass, the material around the focal region absorbs the light energy through the nonlinear photoionization and avalanche ionization [5-9]. Following light absorption, the photoexcited electrons release their energies to the lattice as heat, emit light, or become trapped in metastable states 


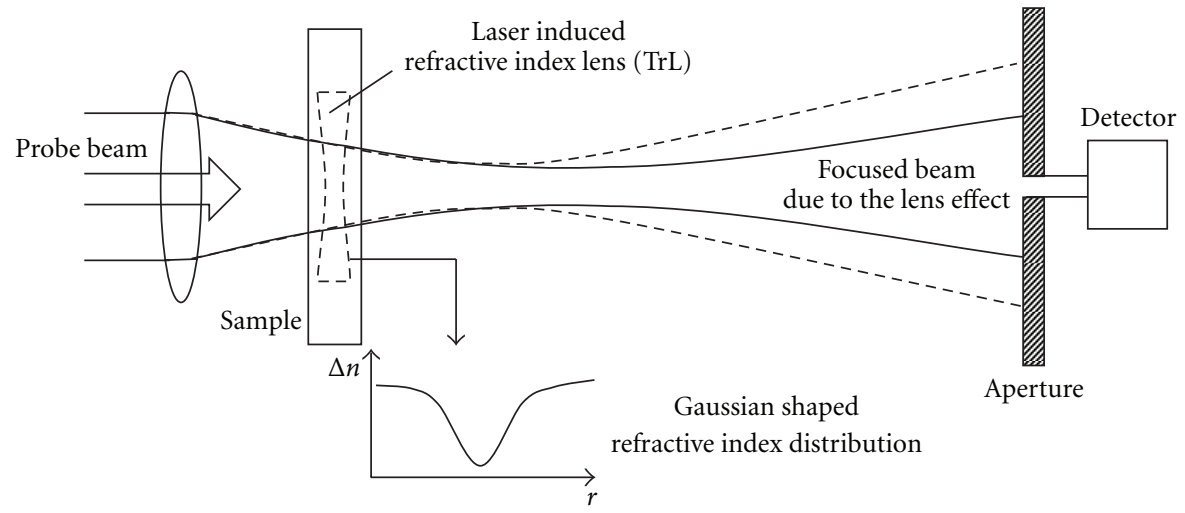

(a)

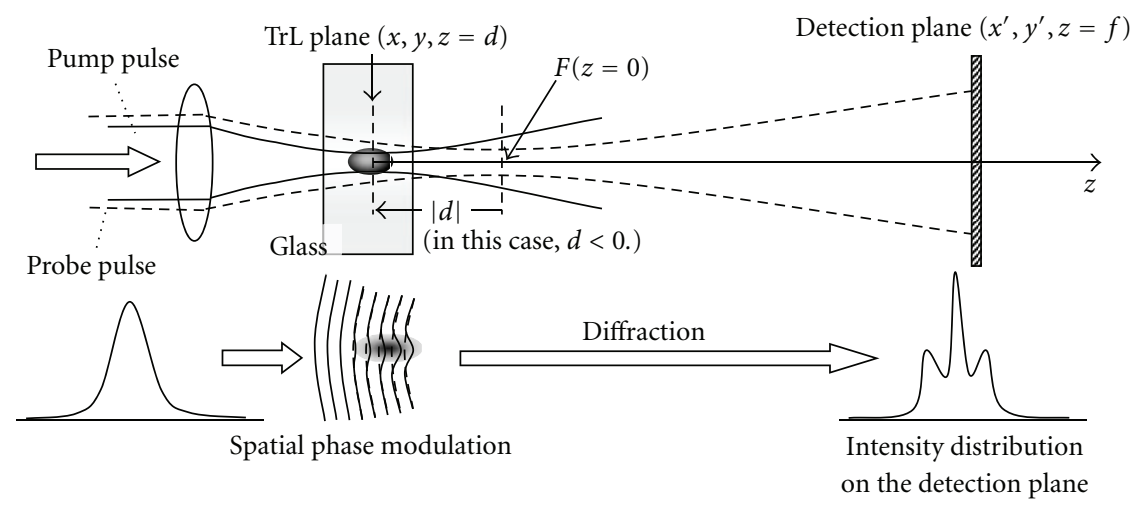

(b)

Figure 1: (a) Principle of the TrL method. (b) TrL method for the observation of fs laser-induced structural change inside a glass. In the lower picture, the deformation process of the beam profile is illustrated.

[28]. The energy transfer from the hot electrons to the lattice results in elevation of temperature in the photoexcited region. The temperature change and the electronic state change should induce the refractive index change; that is, the $\operatorname{TrL}$ is created. To detect this lens, a probe beam is introduced into the photoexcited region. To calculate the intensity distribution on the detection plane, we have to consider the light-matter interaction in the TrL region. During the propagation of the probe beam through the TrL region, the wavefront of the light is modulated due to the refractive index distribution. This is illustrated schematically in Figure 1(b). The modulation of the wavefront corresponds to the modulation of the spatial phase distribution of the probe beam. The phase modulated beam alters its spatial intensity distribution due to diffraction after the propagation $[27,29]$. We can regard the lens effect as the intensity distribution change due to diffraction.

2.3. Calculation Method for Analysis of a TrL Signal. The intensity distribution of the probe light on the detection plane can be calculated by taking into account the spatial phase modulation and the diffraction of the probe light by the $\operatorname{TrL}$ region. The coordinates for the calculation are illustrated in Figure 1(b). The probe beam, which propagates along the $z$-axis, is focused by a lens (the focal length is $f_{\text {obj }}$ ). The focus of the probe beam without a pump beam is located at the position $z=0$. The photoexcited region is located at $z=d$. We call $d$ a "focal mismatch", which is an important factor in determining the TrL signal intensity. The converging probe beam passes through the TrL, and the intensity of the probe beam is detected at $z=f$. The sign of $d$ is very important for interpreting the origin of a TrL signal. In this study, we define that $d$ is negative when the photoexcited region is located behind the focus of the probe beam (the same situation as Figure 1(b)). In this case, if the TrL is convex lens (i.e., positive refractive index change), the probe beam is focused at a shorter distance than that without the TrL effect, so that the probe beam expands at a far field for the detection and the light intensity through the aperture decreases. If the $\mathrm{TrL}$ is a concave type, the light intensity increases (Figure 1(a)) at the detection plane. If $d$ is positive, the light intensity change becomes opposite to the above.

According to the Fresnel diffraction theory [29], the electric field of the probe beam on the detection plane, $E_{\text {SIG }}(X, Y)$, can be described by a convolution of the electric field just after the $\operatorname{TrL}$ region $\left[E_{1}(x, y)\right]$ and the propagation function $\left[g_{z}(x, y)\right]$ :

$$
E_{\mathrm{SIG}}(X, Y)=E_{1}(x, y) \otimes g_{z_{0}}(x, y),
$$




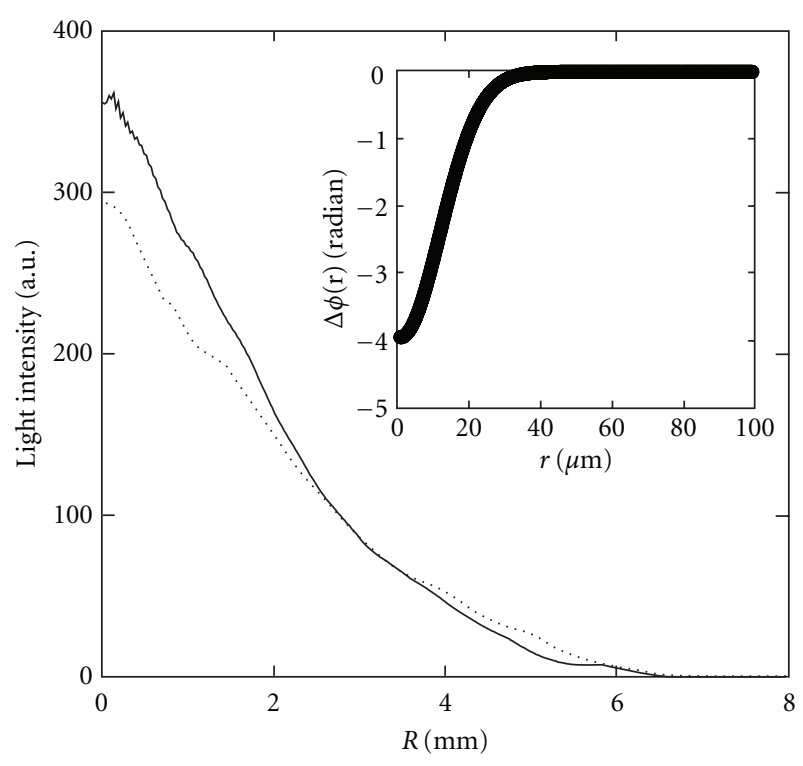

Figure 2: Intensity profile of a probe beam. The solid line is the radian distribution of light intensity without lens effect, and the dotted line is that simulated for the phase distribution shown in the inset.

$$
g_{z}(x, y)=\frac{1}{j \lambda z} \exp \left\{j \pi\left[z+\frac{x^{2}+y^{2}}{\lambda z}\right]\right\}
$$

where $z_{0}$ is the distance between the TrL region and the detection plane (i.e., $z_{0}=f-d$ ), and we assume that the TrL experimental configuration satisfies the Fresnel degree of approximation. In (1) and (2), $\lambda$ is the wavelength of the probe beam, and $\otimes$ denotes the convolution integral. Assuming that there is no transient light absorption, $E_{1}(x, y)$ can be written using the electric field of the probe beam just behind the $\operatorname{TrL}$ region $\left[E_{0}(x, y)\right]$ and the phase modulation due to the $\operatorname{TrL}[\Delta \phi(x, y)]$ as follows:

$$
E_{1}(x, y)=E_{0}(x, y) \exp \{j \Delta \phi(x, y)\} .
$$

Substituting (3) into (1), we can express $E_{\text {SIG }}(X, Y)$ using $E_{0}(x, y)$ :

$$
\begin{aligned}
E_{\mathrm{SIG}}(X, Y)= & \frac{1}{j \lambda z_{0}} \exp \left(j \frac{\pi}{\lambda}\left(\frac{2 z_{0}^{2}+X^{2}+Y^{2}}{z_{0}}\right)\right) \\
& \iint E_{0}(x, y) \exp \left\{j\left(\Delta \phi(x, y)+\pi \frac{x^{2}+y^{2}}{\lambda z_{0}}\right)\right\} \\
& \times \exp \left\{-j 2 \pi \frac{X x+Y y}{\lambda z_{0}}\right\} d x d y .
\end{aligned}
$$

The integral in (4) is the Fourier transform to Fourier space of $\left[f_{X}=2 \pi X /\left(\lambda z_{0}\right), f_{Y}=2 \pi Y /\left(\lambda z_{0}\right)\right]$. In the TrL method, $E_{0}(x, y)$ and $\Delta \phi(x, y)$ are cylindrically symmetric along the beam axis. Therefore, (4) can be written using only a radial variable from the $z$ axis. When a function is circularly symmetric, its Fourier transform can be expressed by an integral with only radial variation (Hankel transform) as follows:

$$
\begin{aligned}
E_{\mathrm{SIG}}(R)= & \frac{2 \pi}{j \lambda z_{0}} \exp \left(j \frac{\pi}{\lambda}\left(\frac{2 z_{0}^{2}+R^{2}}{z_{0}}\right)\right) \\
& \times \int_{0}^{\infty} E_{0}(r) \exp \left\{j\left(\Delta \phi(r)+\pi \frac{r^{2}}{\lambda z_{0}}\right)\right\} \\
& \times J_{0}\left(2 \pi \frac{r R}{\lambda z_{0}}\right) r d r
\end{aligned}
$$

where $r$ and $R$ are the distance from the beam axis, given, respectively, by $r=\left(x^{2}+y^{2}\right)^{1 / 2}$ and $R=\left(X^{2}+Y^{2}\right)^{1 / 2}$, and $J_{0}(x)$ is the 0th order Bessel function. Therefore, the light intensity distribution on the detection plane, $I_{\mathrm{SIG}}(R)$, is given by

$$
\begin{aligned}
I_{\mathrm{SIG}}(R)=\varepsilon_{0} \mid & \frac{2 \pi}{\lambda z_{0}} \int_{0}^{\infty} E_{0}(r) \exp \left\{j\left(\Delta \phi(r)+\pi \frac{r^{2}}{\lambda z_{0}}\right)\right\} \\
& \times\left. J_{0}\left(2 \pi \frac{r R}{\lambda z_{0}}\right) r d r\right|^{2} .
\end{aligned}
$$

In order to calculate $I_{\mathrm{SIG}}(R)$ using (6), we need to know $E_{0}(x, y)$. However, we cannot easily measure this quantity experimentally, because it includes both the phase and intensity of the beam in the small region inside a material. Therefore, we estimated $E_{0}(x, y)$ by using the probe beam profile without $\operatorname{TrL}\left[I_{\mathrm{REF}}(X, Y)\right]$ as follows. When there is no $\operatorname{TrL}$ (i.e., no pump pulse), the phase variation function is uniform throughout the entire region $[\Delta \phi(x, y)=0]$. In this case, the electric field of the probe beam at the detection plane $\left[E_{\mathrm{REF}}(X, Y)\right]$ can be written as

$$
E_{\mathrm{REF}}(X, Y)=\varepsilon^{1 / 2}\left\{I_{\mathrm{REF}}(X, Y)\right\}^{1 / 2} \exp \left[\frac{j \pi\left(X^{2}+Y^{2}\right)}{\lambda f}\right] .
$$

$E_{0}(x, y)$ can be calculated by deconvolution of (4) by substituting $\Delta \phi(x, y)=0$ and replacing $E_{\mathrm{SIG}}(X, Y)$ by $E_{\mathrm{REF}}(X, Y)$ :

$$
\begin{aligned}
E_{0}(x, y)= & \frac{1}{j \lambda z_{0}} \iint E_{\mathrm{REF}}(X, Y) \\
& \exp \left\{j \pi \frac{z_{0}^{2}+(X-x)^{2}+(Y-y)^{2}}{\lambda z_{0}}\right\} d X d Y .
\end{aligned}
$$

Because $E_{0}(x, y)$ is circularly symmetric around the beam axis, (8) can be rewritten by Hankel transform:

$$
\begin{aligned}
E_{0}(r)= & -\frac{1}{j \lambda z_{0}} \exp \left\{j \pi \frac{2 z_{0}^{2}+r^{2}}{\lambda z_{0}}\right\} \\
& \times \iint E_{\mathrm{REF}}(R) \exp \left(-j \pi \frac{R^{2}}{\lambda z_{0}}\right) J_{0}\left(2 \pi \frac{r R}{\lambda z_{0}}\right) R d R .
\end{aligned}
$$


A typical example of $I_{\mathrm{REF}}(R)$ experimentally measured in this study is shown in Figure 2 (dotted line).

For example, the TrL image was calculated by the following phase variation function:

$$
\Delta \phi(r)=-4.0 \exp \left[-\left(\frac{r}{16 \mu \mathrm{m}}\right)^{2}\right]
$$

with $d=-0.06 \mathrm{~mm}, f_{0}=30 \mathrm{~mm}$, and $\lambda=400 \mathrm{~nm}$, the values used for the experiments in this study. This phase distribution is shown in the inset of Figure 2. In this case, the light intensity of the calculated beam (a solid line in Figure 2) at the central region is about 1.2 times larger than that of the reference probe beam. The result is consistent with the intensity change calculated previously using geometrical optics [27].

The $\operatorname{TrL}$ signal intensity $\left(I_{\mathrm{TrL}}\right)$ is defined as the light intensity at the central region of the probe beam (transmittance through an aperture). Since the TrL signal is usually normalized to the signal intensity without a pump pulse, $I_{\mathrm{TrL}}$ is calculated by

$$
I_{\mathrm{TrL}}=\frac{\int_{0}^{a} I_{\mathrm{SIG}}(R) R d R}{\int_{0}^{a} I_{\mathrm{REF}}(R) R d R},
$$

where $a$ is a radius of the aperture.

The expression for $\Delta \phi(r)$ depends on the dynamics [19-21, 27]. For example, the expression for $\Delta \phi(r)$ in the thermal diffusion process is simple, since it can be expressed analytically by solving a diffusion function and is Gaussian shaped $[21,27]$. On the other hand, for the process of elastic relaxation, $\Delta \phi(r)$ is more complex, because it cannot be expressed analytically $[19,20]$. It must be obtained according to the numerical simulation of elastic dynamics. The details for each process will be described in the following sections.

\section{Observation of Thermoelastic Relaxation}

3.1. TrL Measurement in the ps-ns Time Range. For observation of the $\operatorname{TrL}$ signals in the ps-ns time range, an fs laser pulse was used as a pump and probe beam. The experimental setup is illustrated schematically in Figure 3(a). The fs laser pulse from a Ti:sapphire laser with a regenerative amplifier ( $\lambda=800 \mathrm{~nm}, 120 \mathrm{fs}$; Mira-Legend, Coherent Inc.) was used for this experiment. The laser pulse was split into two by a beam splitter. One of the beams was used as a pump pulse. It was focused inside a glass sample using a microscope objective lens $(20 \times$, NA $=0.45)$, and photoexcitation was induced in the focal region. The other beam was passed through a BBO crystal to obtain the second harmonic and this second harmonic light was used as a probe pulse. The probe beam was passed through an optical delay line, which controlled the time delay from $-500 \mathrm{ps}$ to $4500 \mathrm{ps}$, and focused inside the glass sample with the same objective lens. The focal position of the probe beam was controlled by changing the distance between two lenses, L1 and L2. The probe beam after passing through the photoexcited region was collimated by the lens L3. After separation from the pump pulse by a prism, the probe beam was imaged on a charge coupled device (CCD) camera. The glass sample (silica or soda-lime glass; VIOSIL-SQ, ShinEtsu QUARTS and S1225, Matsunami) was placed on a translation stage. During measurement of a TrL signal, the glass sample was translated at about $10 \mathrm{~mm} / \mathrm{s}$ to avoid multiple photoexcitations at the same position.

The images of the probe pulse at different time delays after the photoexcitation of a sodalime glass are shown in Figure 4(a). Typically, the focal mismatch was $d=-0.06 \mathrm{~mm}$ and the energy of a pump pulse was $0.3 \mu \mathrm{J}$. At $-20 \mathrm{ps}$, the intensity distribution was Gaussian. At 0 ps, the spatial profile of the probe beam changed suddenly. This means that the lens effect appeared as a result of the photoexcitation. There was a sudden change in the beam pattern again at 1 ps. After $1 \mathrm{ps}$, the beam pattern changed continuously until $2 \mathrm{~ns}$. The temporal profile of TrL signal is shown in Figure 4(b). At 0 ps, the signal intensity decreased and recovered after 1 ps. The spike-shaped signal change should be attributed to interactions between the glass and the light field, such as the optical Kerr effect $[23,24]$ and electronhole plasma formation $[5,7]$, because the duration was as short as that of the pump pulse [28]. While the optical Kerr effect induces a positive refractive index, the electronhole plasma contributes negatively to the refractive index change [28]. Because the negative signal change at $d<0$ means the positive refractive index change, the dominant origin of the signal at 0 ps should be the optical Kerr effect. After the spike-shaped change, the signal decayed slightly over several ps. Although the signal intensity recovered to that before the photoexcitation, the beam pattern in this time range was completely different from that before the pump pulse. Hence, it is apparent that the refractive index distribution in the photoexcited region changed due to temperature elevation, formation of electron-hole plasma, and local structural change in the glass network. In the longer time range, the signal oscillated several times until 2 ns. The origin of this damping oscillation is explained as follows.

To explain the damping oscillation in the $\operatorname{TrL}$ signal, we simulated the material response in the confined region after the photoexcitation. Because sudden temperature elevation should occur in the photoexcited region, a large thermal stress should be generated within that region. Therefore, we simulated the response of the glass (based on elastic dynamics) to sudden generation of a thermal stress. The equation used in the simulation $[30,31]$ is the timedependent elastic equation for an isotropic material given by

$$
\begin{aligned}
\rho \frac{\partial^{2} \mathbf{u}}{\partial t^{2}}= & \frac{Y}{2(1+\sigma)} \nabla^{2} \mathbf{u}+\frac{Y}{2(1+\sigma)(1-2 \sigma)} \\
& \times \nabla(\nabla \cdot \mathbf{u})-\frac{Y \beta}{3(1-2 \sigma)} \nabla\{\Delta T(t, x, y, z)\},
\end{aligned}
$$

where $\rho$ is the density, $\mathbf{u}$ is displacement vector, $Y$ is the Young's modulus, $\sigma$ is the Poisson's ratio, $\beta$ is the thermal expansion coefficient, and $\Delta T(t, x, y, z)$ is the temperature distribution change due to the photoexcitation. For simplicity, we assumed that a cylindrical shaped temperature 


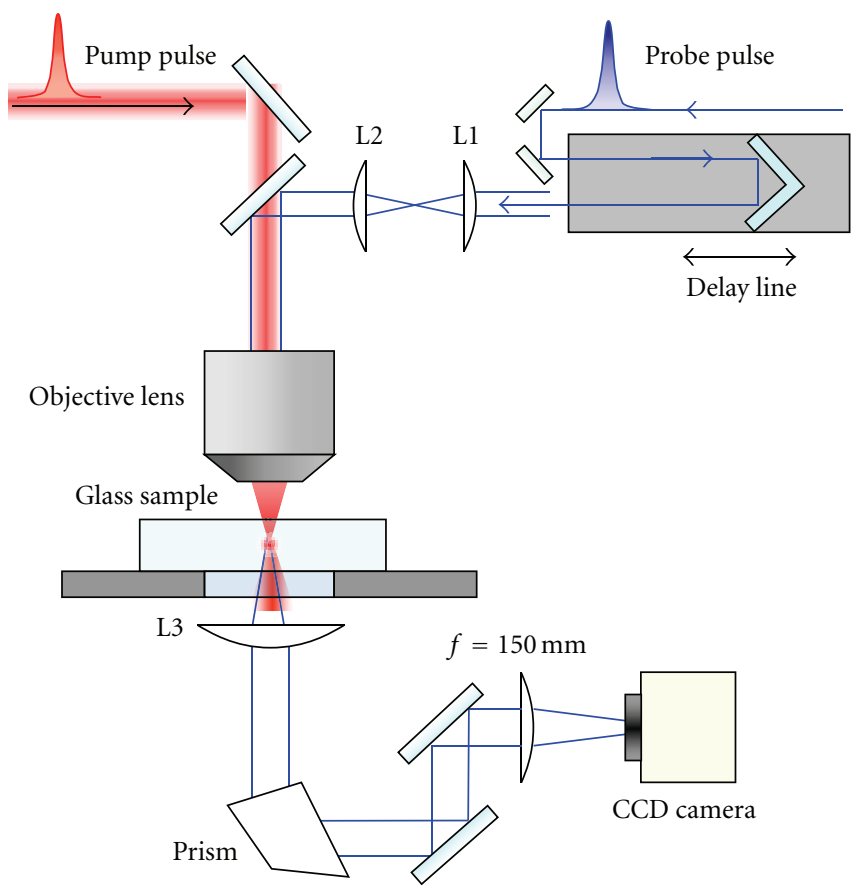

(a)

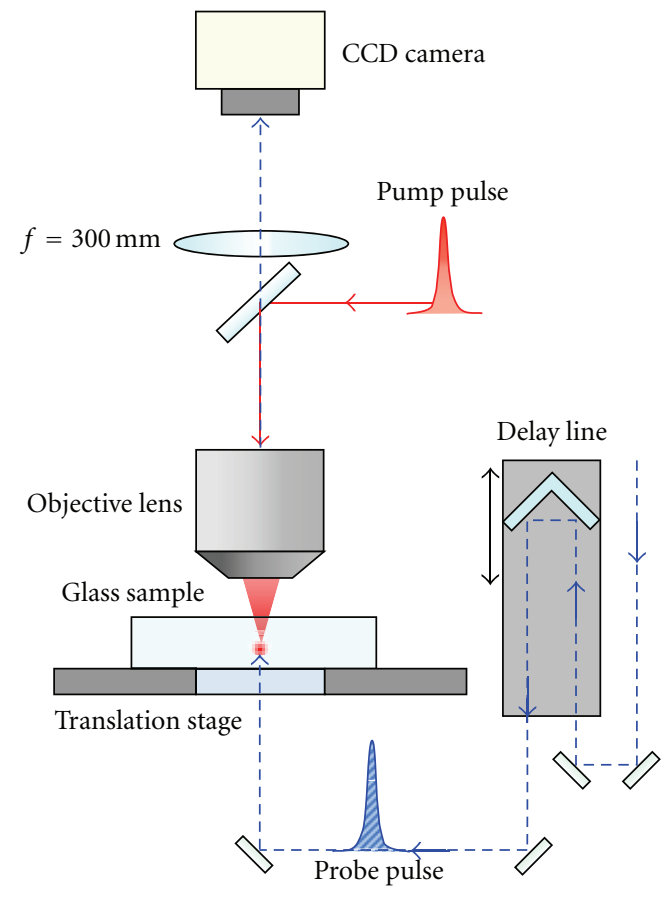

(b)

Figure 3: Experimental setups for (a) TrL method and (b) time-resolved transmission imaging for observing the dynamics after irradiation of a glass with a focused fs laser pulse. OL is an objective lens, DM is a dichroic mirror, and L1, L2, and L3 are lenses.

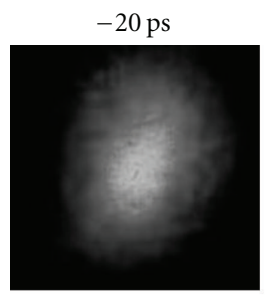

100 ps

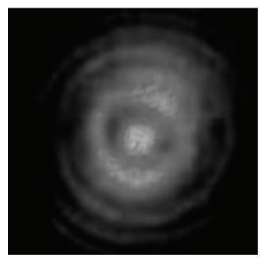

1200 ps

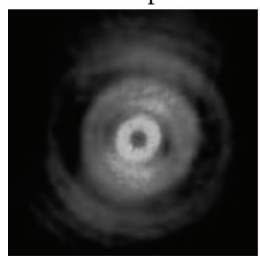

0 ps

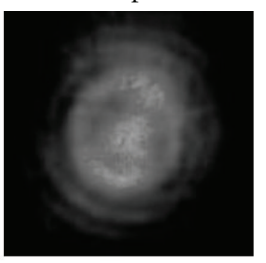

400 ps
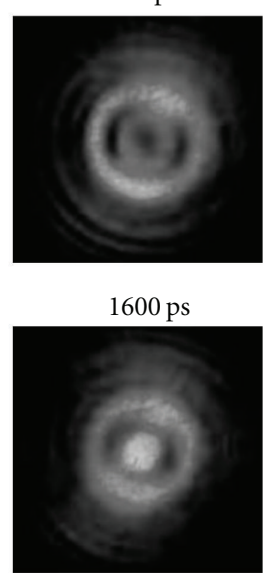

(a)

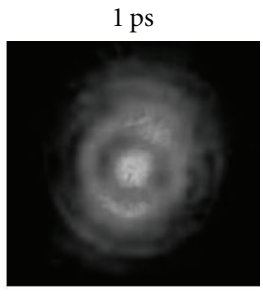

800 ps

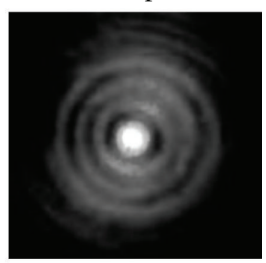

2 ns

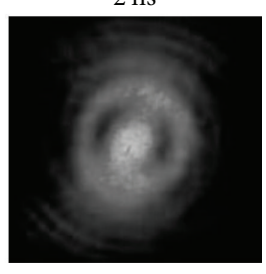

.

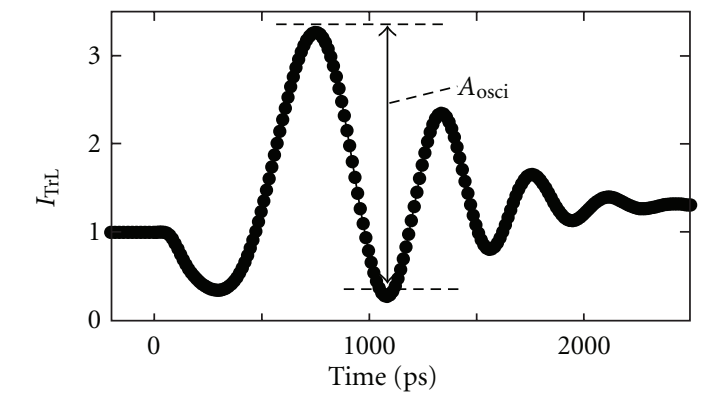

(c)

Figure 4: (a) TrL images at different time-delays and $d=-0.06 \mathrm{~mm}$. The pump energy was $0.25 \mu \mathrm{J} /$ pulse. (b) The TrL signal measured under the same conditions as for (a). (c) The TrL signal simulated using pressure wave propagation. 


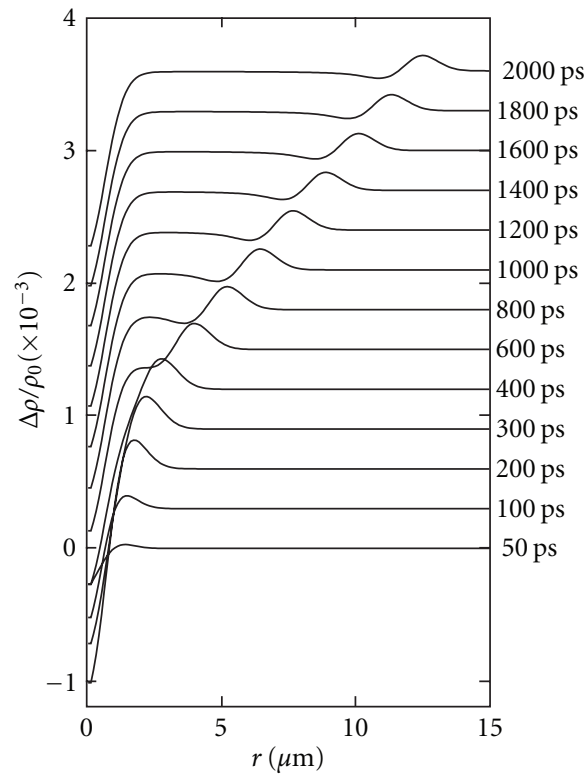

FIgURE 5: Temporal evolution of the simulated density distribution change after the temperature increase of $1000 \mathrm{~K}$ in 1 ps.

distribution was created after the photoexcitation. Therefore, the temperature distribution change was expressed by

$$
\begin{aligned}
\Delta T(t, x, y, z)= & \Delta T_{0} * \exp \left\{-\frac{\left(x^{2}+y^{2}\right)}{\left(w_{\mathrm{th}} / 2\right)^{2}}\right\} \\
& * H(t)\left(\frac{-l_{\mathrm{th}}}{2} \leq z \leq \frac{l_{\mathrm{th}}}{2}\right),
\end{aligned}
$$

where $\Delta T_{0}$ is the maximum temperature change, $w_{\text {th }}$ is the diameter of the photoexcited area, $l_{\text {th }}$ is the length of the temperature distribution along the beam axis, and $H(t)$ is the Heaviside step function of time. It is reasonable to consider that the temperature change is expressed by the step function, because the temperature elevation by an fs laser pulse occurs much faster than the elastic response time [28]. The temporal evolution of the calculated density distribution change is shown in Figure 5. The density change was plotted against the distance from the center. In this calculation, we used $\Delta T_{0}=1000 \mathrm{~K}, w_{\text {th }}=2.0 \mu \mathrm{m}$, and the elastic constants for sodalime glass, $Y=72 \mathrm{GPa}, \sigma=0.29, \rho=2.54 \mathrm{~g} / \mathrm{cm}^{3}$, and $\beta=3.8 \times 10^{-6} \mathrm{~K}^{-1}$ [32]. The density in the central region decreases gradually due to thermal expansion. At the same time, the density in the surrounding region begins to increase, because the material in the surrounding region is compressed by the thermal expansion in the central region. The higher density region propagates away from the center and gradually outwards. The velocity of the propagation is about $6 \mu \mathrm{m} / \mathrm{ns}$, which corresponds to that of the longitudinal sound wave. This propagating wave is a pressure wave or stress wave, which is generated as a result of the relaxation of the thermal stress.
The $\operatorname{TrL}$ signal was calculated using the simulated density distribution change $\Delta \rho(t, r)$. The time-dependent phase variation function $\Delta \phi(t, r)$ is expressed by

$$
\Delta \phi_{\mathrm{PW}}(t, r)=\frac{\pi\left(n_{0}^{4}-1\right) \Delta \rho(t, r)}{2 n_{0} \rho_{0} \lambda} l_{\mathrm{th}},
$$

which can be obtained from the Lorentz-Lorenz relation [19, 29]. The calculated TrL signal is shown in Figure 4(c). The signal reproduced the damping oscillation well. Therefore, we concluded that the damping oscillation originated from the propagation of a pressure wave.

Here, it should be noted that the expressions of the temperature distribution by (13) and the phase distribution by (14) were based on a rough model; that is, in reality, the temperature is not constant in the $z$ direction and the pressure wave propagates also in the $z$ direction. To check the validity of this model, we simulated the $\operatorname{TrL}$ signals by calculating the pressure wave generation using a spherically symmetric temperature distribution. We found that the TrL signal was almost the same as that obtained by (13) and (14). Therefore, the $z$ dependence of the temperature distribution may not be so important for discussing the TrL signal.

In the simulation, the $\operatorname{TrL}$ signal was calculated using $\Delta \phi(t, r)$ obtained from the simulation of a laser-induced pressure wave. In fact, it is possible to determine $\Delta \phi(t, r)$ by analyzing the $\operatorname{TrL}$ images without the thermoelastic simulation. In Section 2, we showed that the TrL images can be expressed by (6). Therefore, if the intensity profile of the TrL image is fitted by (6), we can determine $\Delta \phi(t, r)$. We applied the least-squares approach for fitting of the TrL image [19]. The fitted intensity profiles at different timedelays and the corresponding $\Delta \phi(t, r)$ curves are shown in Figures 6(a) and 6(b), respectively. Figure 6(a) shows that the radial intensity distribution in the $\operatorname{TrL}$ images could be fitted by (6). The temporal evolution of the phase distribution represents the generation and propagation of a pressure wave. This analysis confirms that the main origin of the TrL signal should be a laser-induced pressure wave.

We can estimate the pressure elevation in the photoexcited region from the phase change obtained in this analysis. The phase change at the pressure wave in Figure 6(b) was about $0.3 \mathrm{rad}$. The density change that induced the phase change of $0.3 \mathrm{rad}$ can be estimated using (14). To estimate the density change, we need $l_{\text {th }}$, the length of the phase change in the beam propagation direction. We observed the length of the structural change in the beam propagation direction was about $20 \mu \mathrm{m}$. Therefore, we assumed $l_{z}=20 \mu \mathrm{m}$. Using this value, the density change at the center of the pressure wave was calculated to be $\Delta \rho / \rho_{0}=1.1 \times 10^{-3}$. To induce this density change, the pressure in the pressure wave must be $63 \mathrm{MPa}$, which can be calculated by

$$
\Delta P=\frac{Y}{3(1-2 \sigma)}\left(\frac{\Delta \rho}{\rho_{0}}\right) .
$$

Based on the thermoelastic simulation shown in Figure 5, the magnitude of the density decrease at the center is about 7 times larger than that of the density increase in the pressure 


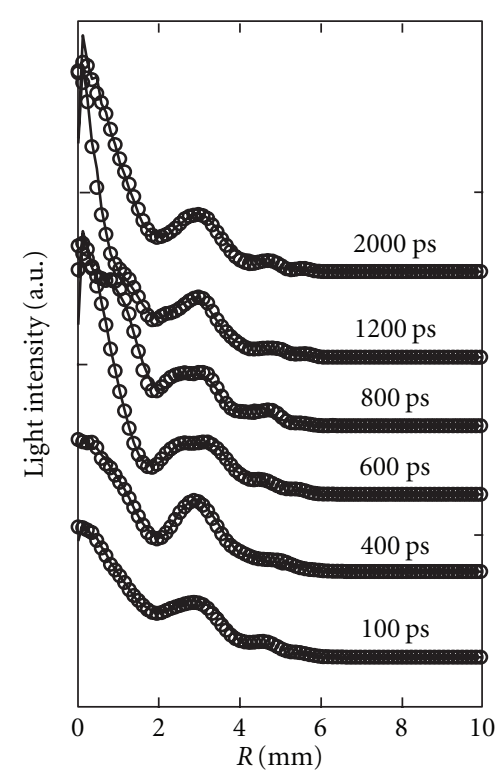

(a)

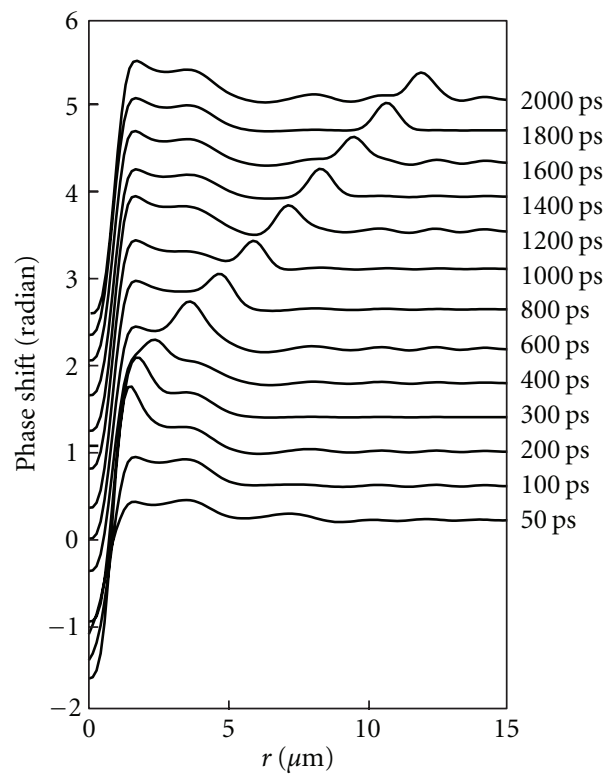

(b)

Figure 6: (a) Radian distribution of light intensity in the TrL images and their fits by (6). (b) The temporal evolution of the phase distributions obtained from the fitting.

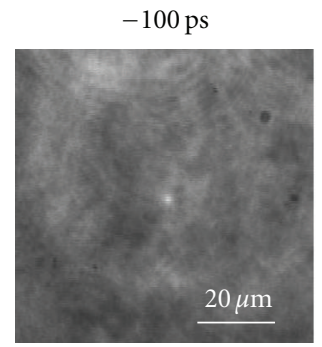

$100 \mathrm{ps}$

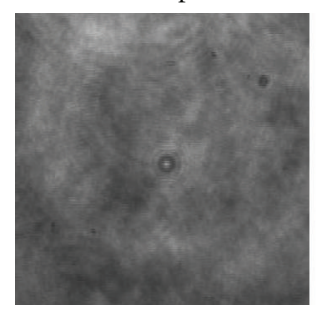

$1000 \mathrm{ps}$

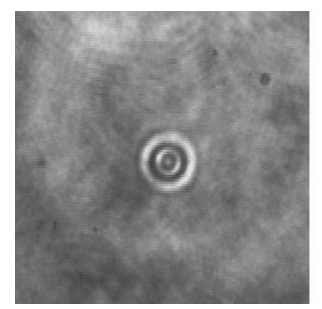

0 ps

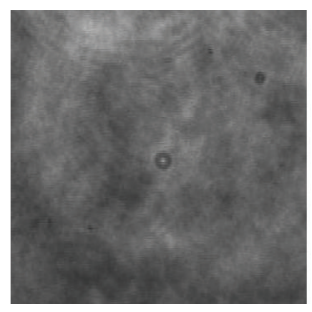

200 ps

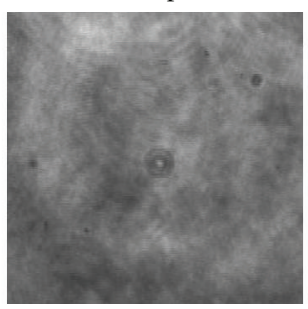

2000 ps

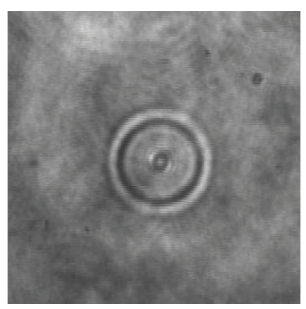

$1 \mathrm{ps}$

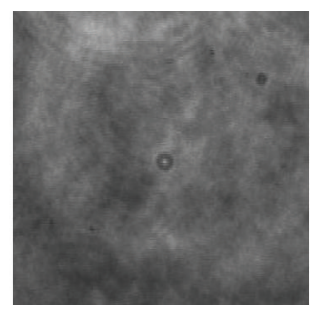

400 ps

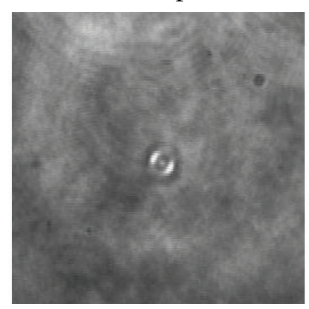

3000 ps

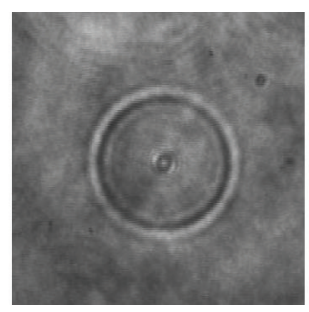

(a)

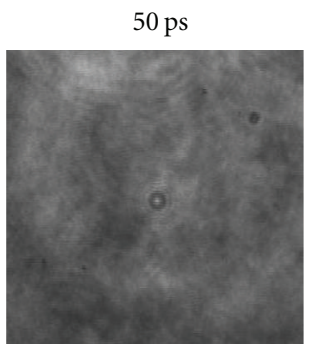

$600 \mathrm{ps}$

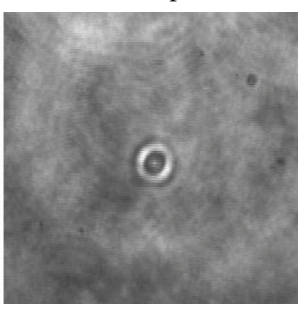

4000 ps
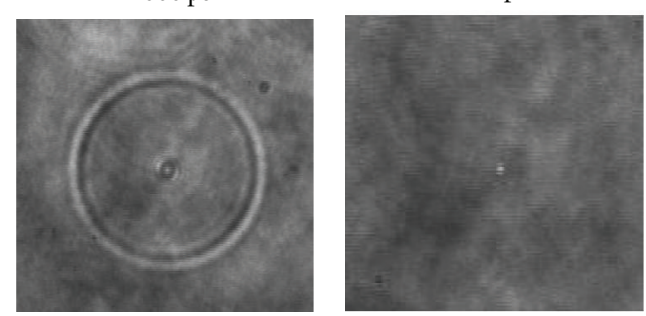

(b)

Figure 7: Time-resolved light transmission imaging (a) $5 \mu \mathrm{J} /$ pulse and (b) $0.2 \mu \mathrm{J} /$ pulse. 
wave. Therefore, the density decrease of $0.8 \%$ and about $400 \mathrm{MPa}$ pressure increase should occur at the center of the photoexcited region after photoexcitation of sodalime glass by a $0.2 \mu \mathrm{J} 100 \mathrm{fs}$ laser pulse. The roughly estimated temperature increase at the photoexcited region is about $2000 \mathrm{~K}$, using a thermal expansion coefficient, $\beta=3.8 \times$ $10^{-6}$. Because the estimated pressure increase is much lower than that at which plastic deformation starts (6-9 GPa) [33], both the temperature and pressure increases should affect the structural change in the photoexcited region.

3.2. Time-Resolved Transmission Imaging. A conventional method for observing the morphology change in laser processing is time-resolved light transmission imaging [9, $11,34,35]$. This method has been used by a number of researchers to observe a laser-induced shock wave inside liquids. For example, Vogel et al. studied the effect of pulse width on the laser-induced shock wave and subsequent generation of a cavitation bubble in water [11,34]. Glezer et al. observed the dynamics of fs laser-induced breakdown in water [35]. The same method can be applied to observation of fs laser-induced structural change inside glasses. Here, we show the time-resolved light transmission imaging result, compare it to the TrL method, and discuss the advantages of each method.

The experimental setup used for time-resolved transmission imaging is shown in Figure 3(b). The difference from the TrL method is the detection method of the probe beam. The probe pulse illuminated the photoexcited region from the opposite direction to the pump pulse, and the intensity distribution in the photoexcited region was imaged on a CCD camera.

Figure 7 shows the transmission images at various timedelays after photoexcitation inside a sodalime glass. Before the photoexcitation $(t=-100 \mathrm{ps})$, a small bright spot was observed at the focal point of the pump pulse. This bright spot should be attributed to a plasma emission, because it was observed without a probe pulse and not observed without a pump pulse. At 0 ps, the focal zone of the pump pulse becomes a tiny bright spot surrounded by a dark ring and remained unchanged until several tens of ps. Although a Kerr effect was observed clearly by the TrL method (spike signals at $0 \mathrm{ps}$ in Figure 4), it was not apparent in the transmission imaging. Around 100 ps, the morphology around the photoexcited region started to change gradually and a pressure wave was generated at the rim of the central dark region. At longer time-delays, the circle became larger, which indicates that the pressure wave propagated away from the photoexcited region. The velocity of the pressure wave was about $5.9 \mu \mathrm{m} / \mathrm{ns}$, which is identical to that measured by the TrL method.

The advantage of transmission imaging is that the morphology and the pressure wave propagation can be seen easily without any analysis. However, this method is not suitable for the evaluation of the properties of the pressure wave, because it is difficult to estimate the amplitude of the pressure wave from the light intensity distribution in the images. On the other hand, the amplitude of the pressure wave can be estimated easily from the oscillation amplitude in a TrL signal. In addition, the signal oscillation can be detected at lower pump energy, while the transmission change at lower pump energy is too weak to be observed in the transmission imaging as shown in Figure 7(b). Therefore, the advantages of the TrL method are (i) high sensitivity and (ii) possible quantitative estimation of the amplitude of the pressure wave. These advantages enable us to measure the energy threshold and the pump energy-dependence of the pressure wave generation. The energy threshold and the pump energy-dependence will be shown in the next section.

\subsection{Pump Energy-Dependence of a Pressure Wave. Although} the analysis shown in Figure 6 visualized the profile of a pressure wave, the time-consuming nature of this analysis is a critical problem especially when we have to compare the TrL signals measured under various conditions. It would be preferable that the properties of a pressure wave can be obtained only from a TrL signal . In Figure 4(c), we showed that the oscillating component in the TrL signal (we call this "TrL oscillation") originates from the propagation of a pressure wave. This suggests that the amplitude of the pressure wave can be estimated from that of the TrL oscillation. In our previous study, we showed that the amplitude and damping rate of the TrL oscillation is determined by the amplitude and width of the pressure wave [20]. Therefore, we can determine the threshold of pressure wave generation and pump energydependence of a pressure wave by the measurement of the amplitudes of the TrL oscillations. Here, we show how the threshold and pump energy dependence differ between two different glasses.

We compared the TrL signals of two often-used glasses, a silica glass and sodalime glass. Although the glass forming elements of two glasses are the same $\left(\mathrm{SiO}_{2}\right)$, the properties of the two glasses are quite different [36-38]. For example, the viscosity of silica glass at high temperature is more than four orders of magnitude larger than that of sodalime glass. This is one reason why the production, casting, and polishing of silica glass is much more difficult than that of sodalime glass. Another important difference is the thermal expansion coefficient, which is six times larger for sodalime glass than for silica glass. There is also a difference in the optical band gap: about $3.2 \mathrm{eV}$ for sodalime glass [39] and $7.5 \mathrm{eV}$ for silica glass [6]. The properties of both glasses are summarized in Table 1. We expect that these differences should affect the generation of a pressure wave.

The TrL signals from silica glass and sodalime glass at identical pump energies are shown in Figure 8(a). Clearly, the amplitude of the TrL oscillation of the sodalime glass is larger than that of the silica glass. The time-period of the oscillation of a silica glass is slightly shorter than that of a sodalime glass. This should be due to differences in the sound velocity in the glasses $(5.93 \mathrm{~km} / \mathrm{s}$ in a silica glass and $5.5 \mathrm{~km} / \mathrm{s}$ in a sodalime glass), because the $\operatorname{TrL}$ oscillation is originated from the propagation of a pressure wave. To compare the amplitude of the TrL oscillation, we defined an oscillation amplitude $\left(A_{\text {osci }}\right)$ as shown in Figure $4(\mathrm{c})$, that is, the difference of the signal intensity at 
TABLE 1: Viscosities $\eta[32,37]$, thermal expansion coefficients $\alpha[32,36]$, band gaps $E_{g}[6,39]$, refractive indices $n$ [36], and glass transition temperatures $T_{g}[38]$ of silica glass and sodalime glass [32].

\begin{tabular}{lccccc}
\hline & $\eta(\mathrm{Pa} \cdot \mathrm{s})$ at $600^{\circ} \mathrm{C}$ & $\eta(\mathrm{Pa} \cdot \mathrm{s})$ at $1200^{\circ} \mathrm{C}$ & $\alpha\left(10^{-6} /{ }^{\circ} \mathrm{C}\right)$ & $E_{g}(\mathrm{eV})$ & $n(590 \mathrm{~nm})$ \\
\hline Silica glass & $>10^{16}$ & $\sim 10^{11}$ & 0.6 & 7.5 & 1.46 \\
Sodalime glass & $\sim 10^{11}$ & $\sim 10^{2}$ & 3.8 & $\sim 3.2$ & $\sim 1100$ \\
\hline
\end{tabular}

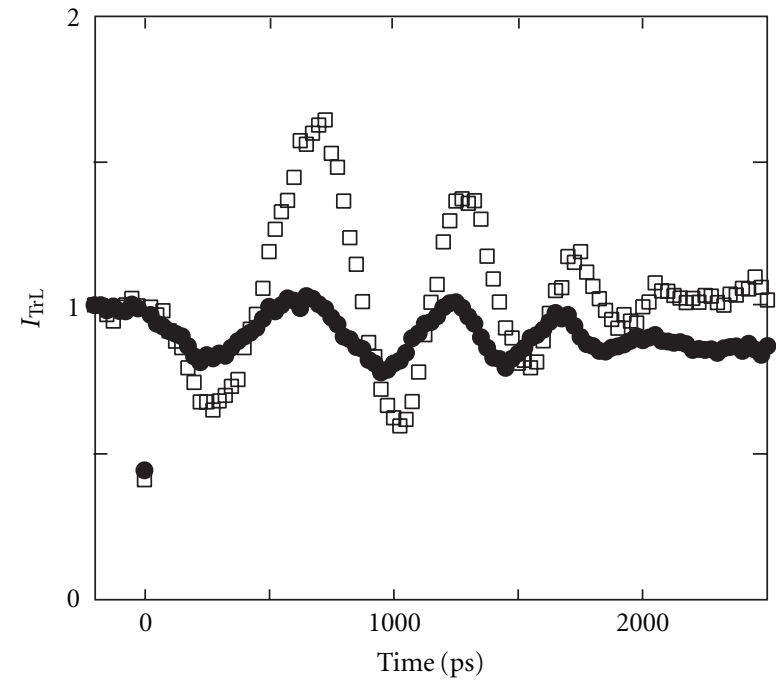

$\square$ Sodalime glass

- Silica glass

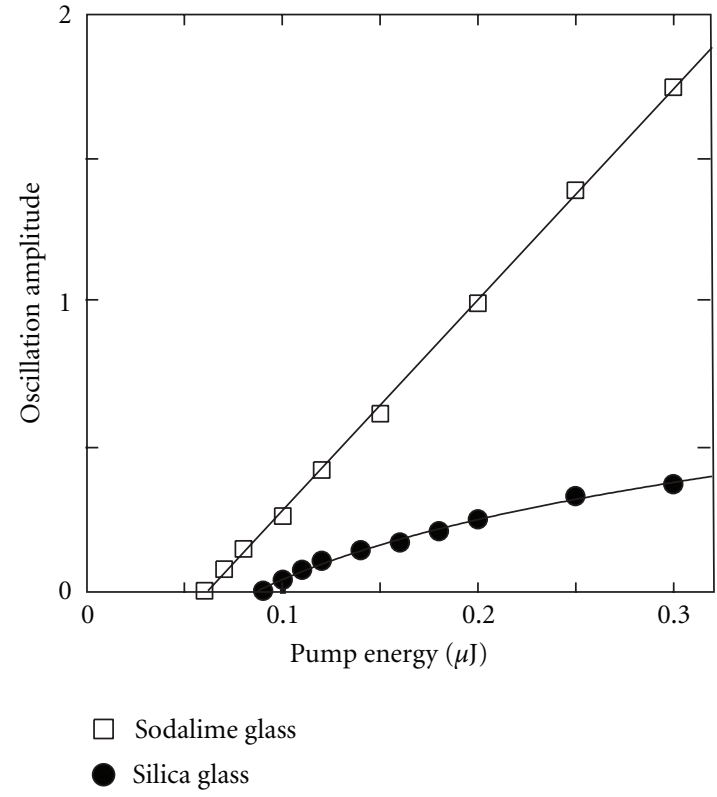

(b)

FiguRE 8: (a) TrL signal of silica glass (closed circles) and sodalime glass (open squares), measured with the same pump energy (0.2 $\mu$ J/pulse). (b) Pump energy dependence of the oscillation amplitude in the TrL signals. Closed circles are silica glass and open squares are sodalime glass.

the first positive peak and that at the second negative peak. The oscillation amplitudes are plotted against the pump energy in Figure 8(b). In both glasses, there were energythresholds of pressure wave generation, and the oscillation amplitude increased with increasing pump energy. The thresholds were about $0.06 \mu \mathrm{J} /$ pulse for the sodalime glass and $0.09 \mu \mathrm{J} /$ pulse for the silica glass. According to the band gaps of these glasses (Table 1), this difference should be attributed to the difference in band gap energies, because three photons are necessary to excite sodalime glass while five photons are required for silica glass. Another possible origin of the difference in the threshold is the glass transition temperature. Because the glass transition temperature of silica glass is about $500^{\circ} \mathrm{C}$ higher than that of sodalime glass, more light energy is needed to deform the silica glass. A further difference is the slope of the oscillation amplitude versus the pump energy; the slope for sodalime glass is more than twice that for silica glass. This difference can be explained by thermal expansion coefficient, because the thermal expansion coefficient of a sodalime glass is about 10 times larger than that of a silica glass. It means that the same temperature increase can induce a 10 times larger density change inside sodalime glass than silica glass.

\section{Observation of Thermal Diffusion}

4.1. TrL Signals in the ns- $\mu$ s Time Range. Although the elastic relaxation was completed within several nanoseconds, the temperature in the photoexcited region should still be high at the longest timedelay used in the $\operatorname{TrL}$ measurement system. This can be confirmed from both the TrL image and the transmission image at the longest time-delay (Figures 4 and 7). In this longer time range, there should be slower dynamics such as thermal diffusion and viscoelastic deformation. Indeed, thermal diffusion is very important for understanding the mechanism of laser processing, because there have been a number of reports stating that the material deformation depends on the repetition rate of laser irradiation, an effect which must be caused by the accumulation of thermal energy. For example, in fs laser processing inside glasses, the morphology induced by the irradiation at a few $\mathrm{kHz}$ is completely different from that at several hundred $\mathrm{kHz}[6,40]$. Since the deformed volume at several $\mathrm{kHz}$ is much larger than the photoexcited volume, the accumulated thermal energies should play an important role in the material deformation. However, little has been reported on the observation of thermal diffusion in fs laser processing. Here, we show the TrL measurement in the time 
range from ns to $\mu$ s and review the investigation of the thermal diffusion process inside a glass.

To observe the dynamics in longer time range, we used a continuous wave (CW) laser beam as a probe beam (an He-Ne laser; $\lambda_{\text {probe }}=633 \mathrm{~nm}$ ). The schematic experimental setup is shown in Figure 9. Pump pulses at $100 \mathrm{~Hz}$ came from a regenerative amplified Er-doped fiber laser (IFRIT; Cyber Laser; $780 \mathrm{~nm}, 220 \mathrm{fs}$ ). The repetition rate of the pump pulses was reduced to $3 \mathrm{~Hz}$ by the mechanical shutter. The pump pulse was focused inside a borosilicate glass sample (Corning 0211). After passing through the photoexcited region, the probe beam was input in a pinhole, and the transmitted light was detected by a photomultiplier (HAMAMATSU; R955), and the time profile of the light intensity was recorded with a digital oscilloscope (Tektronix; TDS 784D). The glass sample was moved about 2 milliseconds after each photoexcitation to avoid multiple excitations at the same position.

Figure 10(a) shows the TrL signals from Corning 0211 with different pump energies. The signal increased for several hundred ns and decayed over several $\mu$ s. The signal intensity became larger with increasing pump energy. Because the thermal diffusion process should be involved in this signal, we simulated the TrL signals using a thermal diffusion model. Under the assumption that the initial temperature distribution is Gaussian shaped and the thermal diffusion constant is independent of time, the temporal evolution of the temperature distribution $[\Delta T(t, r, z)]$ is given by $[21]$

$$
\begin{aligned}
\Delta T(t, r, z)= & \Delta T_{0} \frac{\left(w_{\mathrm{th}} / 2\right)^{2}}{\left(w_{\mathrm{th}} / 2\right)^{2}+4 D_{\mathrm{th}} t} \cdot\left\{\frac{\left(l_{z} / 2\right)^{2}}{\left(l_{z} / 2\right)^{2}+4 D_{\mathrm{th}} t}\right\}^{1 / 2} \\
& \times \exp \left[-\frac{r^{2}}{\left(w_{\mathrm{th}} / 2\right)^{2}+4 D_{\mathrm{th}} t}-\frac{z^{2}}{l_{z}^{2}+4 D_{\mathrm{th}} t}\right],
\end{aligned}
$$

where $t$ is the time after the photoexcitation, $r$ is the distance from the center in the radial direction, $z$ is the position along the beam axis, $\Delta T_{0}$ is the maximum temperature increase, $w_{\text {th }}$ and $l_{z}$ are the radius and longitudinal length of the initial temperature distribution, respectively, and $D_{\text {th }}$ is the thermal diffusivity of the material. Using this equation, the phase variation function due to the temperature distribution can be written as

$$
\begin{aligned}
\Delta \phi_{\mathrm{th}}(t, r) & =\frac{2 \pi \int(d n / d T) \Delta T(t, r, z) d z}{n_{0} \lambda} \\
& =\Delta \phi_{\mathrm{th}} \frac{\left(w_{\mathrm{th}} / 2\right)^{2}}{\left(w_{\mathrm{th}} / 2\right)^{2}+4 D_{\mathrm{th}} t} \exp \left[-\frac{r^{2}}{\left(w_{\mathrm{th}} / 2\right)^{2}+4 D_{\mathrm{th}} t}\right],
\end{aligned}
$$

$$
\Delta \phi_{\mathrm{th}}=\frac{2 \pi^{3 / 2}}{n_{0} \lambda}\left(\frac{d n}{d T}\right) \Delta T_{0} l_{z}
$$

We simulated the TrL signals by substituting (17) into (6) and (11). The fitting parameters were $\Delta \phi_{\text {th }}, w_{\text {th }}$, and $D_{\text {th }}$. The TrL signals simulated with $w_{\text {th }}=2.0 \mu \mathrm{m}$, and $D_{\text {th }}=$ $0.46 \mu \mathrm{m}^{2} \mu \mathrm{s}^{-1}$ were plotted in Figure 10(b). The thermal

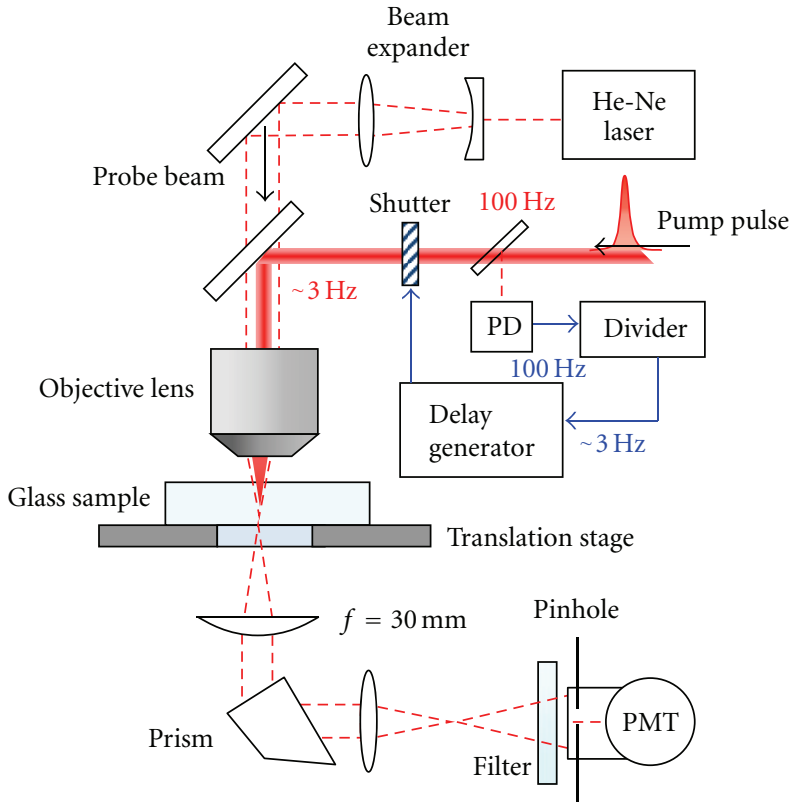

FIGURE 9: Experimental setup for TrL measurement for observing the dynamics in the time range from nano second to micro second.

diffusion constant used in these simulations is the same as that of Corning 0211 at ambient temperature, and the maximum phase changes, $\Delta \phi_{\mathrm{th}}$, were chosen to reproduce the signals well. All the simulated signals reproduced the experimental ones in the time range between $100 \mathrm{~ns}$ and milliseconds very well. It should be noted that the rising and decaying signal can be explained only by thermal diffusion. A qualitative explanation of how thermal diffusion processes lead to the rise and decay is as follows: just after the photoexcitation, the width of the heated region $(\sim 2 \mu \mathrm{m})$ is smaller than that of the probe beam $(>10 \mu \mathrm{m})$. In this case, only the central region of the probe beam is affected by the thermal lens (TL) effect, which results in a weak TrL signal. As the thermal energy diffuses away, the temperature distribution becomes broad; that is, the temperature at the center becomes lower and the width becomes larger. The broadening of the temperature distribution causes a larger effect on the probe beam, so that the signal becomes larger. At longer time (microseconds), the temperature distribution becomes broader than the diameter of the probe beam. After that time, the signal intensity starts to decay.

4.2. Temporal Evolution of Temperature Distribution. Although Figures 10(a) and 10(b) show that the simulated signals reproduced the measured signals at $d=-0.07 \mathrm{~mm}$ very well, the chosen $\Delta \phi_{\text {th }}$ could not reproduce the $\operatorname{TrL}$ signals measured at other $d$ s. This is because $D_{\text {th }}$ and $w_{\text {th }}$ were fixed in the fitting process of the signals at $d=-0.07 \mathrm{~mm}$. Therefore, we searched $\Delta \phi_{\text {th }}, w_{\text {th }}$, and $D_{\text {th }}$ to reproduce the TrL signals at four different $d s$. We found that the TrL signals simulated with $\Delta \phi_{\text {th }}=5.8, w_{\text {th }}=1.7 \mu \mathrm{m}$, and $D_{\text {th }}=0.75 \mu \mathrm{m}^{2} \mu \mathrm{s}^{-1}$ reproduced the measured ones with a pump energy of $0.6 \mu \mathrm{J}$ at four different $d s$ rather 


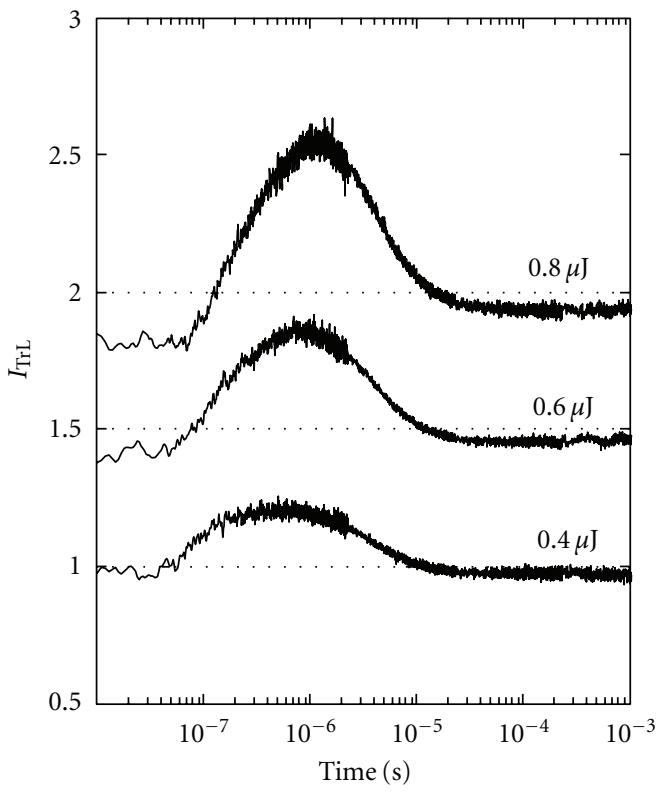

(a)

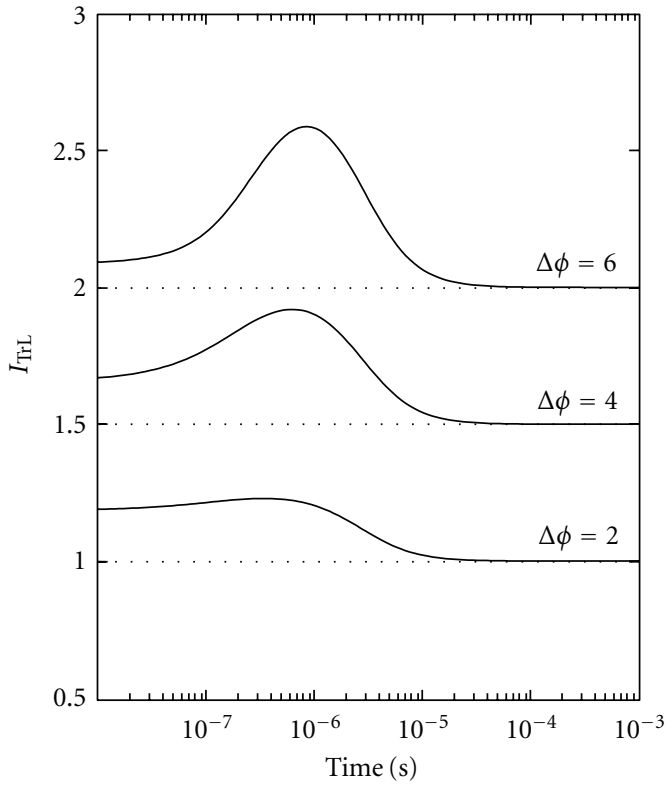

(b)

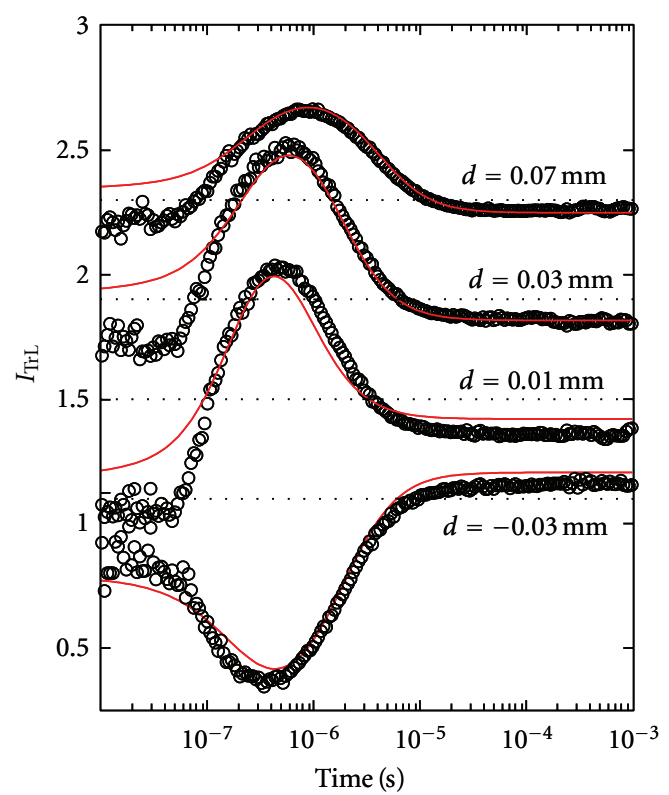

(c)

Figure 10: (a) TrL signals measured with different pump energies. (The signals at $0.6 \mu \mathrm{J}$ and $0.8 \mu \mathrm{J}$ are shifted upward to avoid the overlapping.) (b) Simulated TrL signals to reproduce those in (a). (c) TrL signals measured at different focal position mismatches with a pump energy of $0.6 \mu \mathrm{J} /$ pulse. (The baseline of each signal is shifted to avoid the overlapping.)

well. The simulated and measured signals are shown in Figure 10(c). The assumed length of the $\operatorname{TrL}\left(l_{z}=30 \mu \mathrm{m}\right)$ and the obtained phase change may be large enough for the beam to be diffracted during the propagation in the TrL. This diffraction could affect the analysis of the TrL signal. To estimate the error of the analysis, we simulated the TrL signals with and without diffraction in a TrL by the beam propagation method. We found that the peak of the TrL signal simulated with the diffraction was 30\% larger than that without the diffraction in the case of $l_{z}=50 \mu \mathrm{m}$.
Therefore, the analysis without considering the diffraction effect underestimates the phase change. However, we found that the diffraction effect is less than $3 \%$ in the case of $l_{z}<30 \mu \mathrm{m}$. To discuss the error of the estimation, we have to determine the exact value of $l_{z}$. A more detailed discussion will be presented in future.

We can estimate the temperature increase in the photoexcited region using (18) and $\Delta \phi_{\mathrm{th}}$, which was obtained from the fitting. Assuming $l_{z}=50 \mu \mathrm{m}$ and using $n_{0}=1.5$ and $d n / d T=3.4 \times 10^{-6} \mathrm{~K}^{-1}$ for a borosilicate glass [36], 


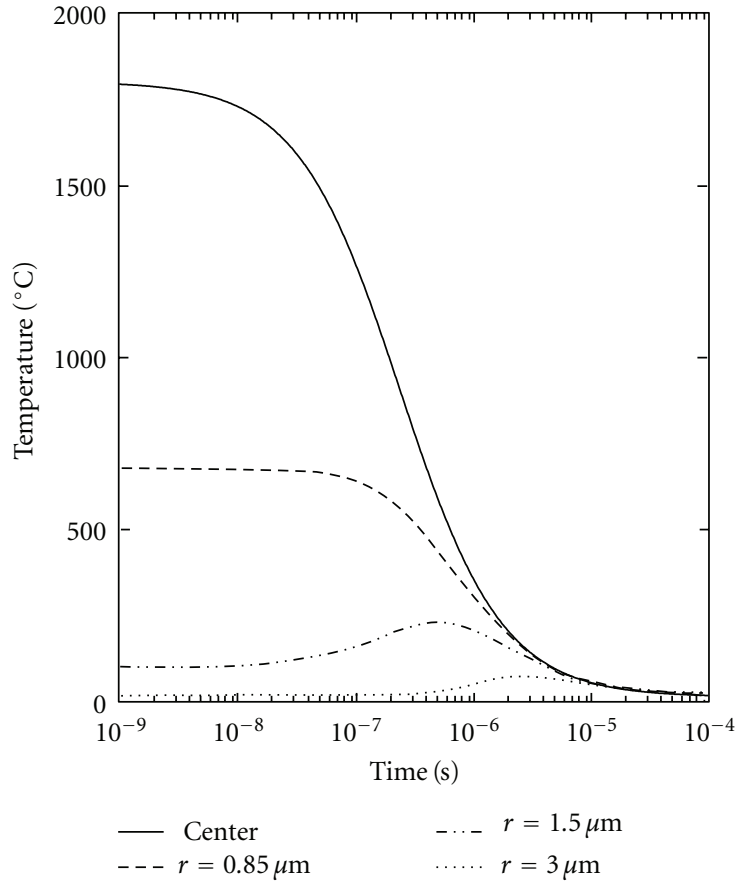

(a)

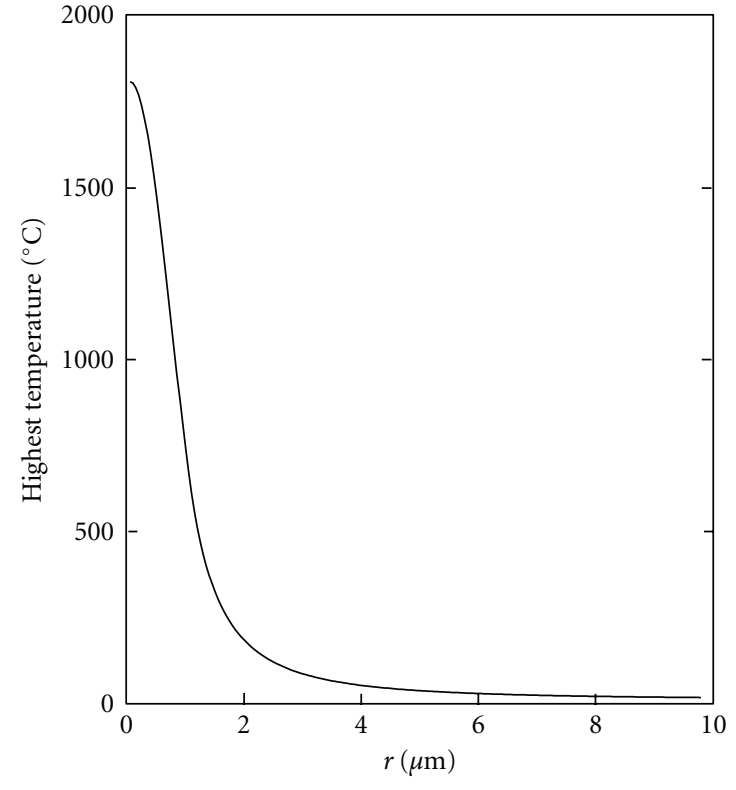

(b)

FIgURE 11: (a) Temporal evolutions of temperature at different positions. They were calculated based on the analyses of the TrL signals measured with a pump energy of $0.6 \mu \mathrm{J} /$ pulse. (b) The highest temperature which the material reaches after the photoexcitation by fs laser pulse of $0.6 \mu \mathrm{J}$.

we obtained $\Delta T_{0} \sim 1790 \mathrm{~K}$. Using this $\Delta T_{0}$ and $D_{\text {th }}$ and $w_{\text {th }}$, we calculated the temporal evolution of the temperature as shown in Figure 11(a). The temperature at the center remains at above $1500^{\circ} \mathrm{C}$ for $100 \mathrm{~ns}$ after the photoexcitation. Due to this high temperature, the glass at the center should be molten, with a viscosity low enough for viscous flow to occur. We now estimate how much viscous flow occurred at the center. Using the viscosity data for Corning 0211 [41] and Fulcher's equation [42], the viscosity at the center in $100 \mathrm{~ns}$ after photoexcitation should be about $10 \mathrm{~Pa} \cdot \mathrm{s}$. According to the Stokes-Einstein (SE) relation [43], the diffusion coefficient of a spherical molecule in a continuum solvent is given by

$$
D_{\mathrm{SE}}=\frac{k T}{6 \pi \eta r},
$$

where $k$ is the Boltzmann constant, $T$ is temperature, $\eta$ is viscosity, and $r$ is the radius of the molecule. We assumed that the minimum radius of the moving component in the molten state is the bond length of $\mathrm{B}-\mathrm{O}(\sim 0.1 \mathrm{~nm})$ [44]. Under this assumption, $D_{\mathrm{SE}} \sim 1.4 \times 10^{-11} \mathrm{~m}^{2} \mathrm{~s}^{-1}$ was obtained. The diffusion length of the molecule with $D_{\mathrm{SE}} \sim 1.4 \times$ $10^{-11} \mathrm{~m}^{2} \mathrm{~s}^{-1}$ in $100 \mathrm{~ns}$ may be calculated to be $1.2 \mathrm{~nm}$. This estimate means that the region undergoing viscous flow in fs-laser processing inside a glass is very small compared to the photoexcited region.

The temporal evolution of the temperature distribution elucidates the highest temperature of the material after the photoexcitation. At $r<w_{\text {th }} / 2$, the temperature is highest just after the photoexcitation. On the other hand, at $r>$ $w_{\text {th }} / 2$, the temperature reaches its highest level during the thermal diffusion process (e.g., $r=1.5 \mu \mathrm{m}$ and $3.0 \mu \mathrm{m}$ in Figure 11(a)). The highest temperatures were plotted as a function of the distance from the center in Figure 11(b). This plot indicates that the temperature at $r>1.0 \mu \mathrm{m}$ does not overcome the glass transition range of the glass. Therefore, the thermal effect of a single irradiation on the surrounding region is very small.

\section{Summary}

We presented the investigation of refractive index dynamics, which reflects density, pressure, and temperature changes, after the photoexcitation by an fs laser pulse inside a glass by the TrL method. In the shorter time range, the pressure wave generation was observed clearly as a damping oscillation in the TrL signal ( $\operatorname{TrL}$ oscillation). This oscillation could be observed with weak excitation energy near the threshold of the structural change. By comparing the time-resolved transmission imaging, the sensitivity of the pressure wave was much higher in the TrL method. In addition, the amplitudes of the pressure wave could be evaluated from the oscillation amplitudes in the TrL signals. Therefore, the TrL method enabled us to investigate the excitation energy dependence of the amplitude of the pressure wave near the threshold and the difference in dynamics between different glasses. In the longer time range, the rise and decay signal was 
observed, which could be explained by a thermal diffusion process. By fitting the signal using a thermal diffusion equation, the temporal evolution of the temperature around the photoexcitation was obtained. From this analysis, we found that the cooling in the photoexcited region was fast enough to prevent large viscous flow in the photoexcited region and the temperature increase in the surrounding region is low not to induce thermal deformation.

The investigation of the refractive index dynamics by the $\operatorname{TrL}$ method showed the characteristic of an fs laser processing inside a glass. The rapid temperature increase by the irradiation of fs laser pulse, which is much faster than the elastic response time of the photoexcited material, generates a strong pressure wave in the elastic relaxation process. In the case of the photoexcitation by a longer laser pulse, much more energy is needed to generate the pressure wave of the same amplitude. This means that the material deformation can be induced with a weaker photoexcitation by the fs laser irradiation. The localized photoexcitation inside a glass by the fs laser pulse should be important for preventing a random deformation due to viscous flow of heated material at high temperature. The localized thermal energy generated by the photoexcitation diffuses out from the photoexcited region so fast. Since the viscosity depends on the temperature, the viscosity of the heated material should increase very fast, with the same rate of the thermal diffusion. The fast increase in viscosity should prevent the viscous flow of the heated material.

\section{Acknowledgments}

One of the authors (M. Terazima) acknowledges Grant-inAid for Scientific Research on Innovative Areas (Research in a proposed research area) (20107003) from the Ministry of Education, Science, Sports and Culture in Japan. This research was also partly supported by the New Energy and Industrial Technology Development Organization (NEDO), the Murata science foundation, and Amada foundation for metal work technology.

\section{References}

[1] K. M. Davis, K. Miura, N. Sugimoto, and K. Hirao, "Writing waveguides in glass with a femtosecond laser," Optics Letters, vol. 21, no. 21, pp. 1729-1731, 1996.

[2] E. N. Glezer, M. Milosavljevic, L. Huang et al., "Threedimensional optical storage inside transparent materials," Optics Letters, vol. 21, no. 24, pp. 2023-2025, 1996.

[3] H.-B. Sun, V. Mizeikis, Y. Xu et al., "Microcavities in polymeric photonic crystals," Applied Physics Letters, vol. 79, no. 1, pp. 13, 2001.

[4] W. Watanabe, T. Toma, K. Yamada, J. Nishii, K. I. Hayashi, and K. Itoh, "Optical seizing and merging of voids in silica glass with infrared femtosecond laser pulses," Optics Letters, vol. 25, no. 22, pp. 1669-1671, 2000.

[5] L. Sudrie, A. Couairon, M. Franco et al., "Femtosecond laserinduced damage and filamentary propagation in fused silica," Physical Review Letters, vol. 89, no. 18, Article ID 186601, 4 pages, 2002.
[6] C. B. Schaffer, J. F. García, and E. Mazur, "Bulk heating of transparent materials using a high-repetition-rate femtosecond laser," Applied Physics A, vol. 76, no. 3, pp. 351-354, 2003.

[7] A. Mermillod-Blondin, I. M. Burakov, Y. P. Meshcheryakov et al., "Flipping the sign of refractive index changes in ultrafast and temporally shaped laser-irradiated borosilicate crown optical glass at high repetition rates," Physical Review B, vol. 77, no. 10, Article ID 104205, 2008.

[8] B. C. Stuart, M. D. Feit, A. M. Rubenchik, B. W. Shore, and M. D. Perry, "Laser-induced damage in dielectrics with nanosecond to subpicosecond pulses," Physical Review Letters, vol. 74, no. 12, pp. 2248-2251, 1995.

[9] K. Sokolowski-Tinten, J. Bialkowski, A. Cavalleri et al., "Transient states of matter during short pulse laser ablation," Physical Review Letters, vol. 81, no. 1, pp. 224-227, 1998.

[10] G. Paltauf and P. E. Dyer, "Photomechanical processes and effects in ablation," Chemical Reviews, vol. 103, no. 2, pp. 487518, 2003.

[11] A. Vogel, J. Noack, G. Hüttman, and G. Paltauf, "Mechanisms of femtosecond laser nanosurgery of cells and tissues," Applied Physics B, vol. 81, no. 8, pp. 1015-1047, 2005.

[12] J. W. Chan, T. R. Huser, S. H. Risbud, and D. M. Krol, "Modification of the fused silica glass network associated with waveguide fabrication using femtosecond laser pulses," Applied Physics A, vol. 76, no. 3, pp. 367-372, 2003.

[13] M. Watanabe, S. Juodkazis, H. B. Sun, S. Matsuo, and H. Misawa, "Luminescence and defect formation by visible and near-infrared irradiation of vitreous silica," Physical Review B, vol. 60, no. 14, pp. 9959-9964, 1999.

[14] L. V. Zhigilei and B. J. Garrison, "Microscopic mechanisms of laser ablation of organic solids in the thermal and stress confinement irradiation regimes," Journal of Applied Physics, vol. 88, no. 3, pp. 1281-1298, 2000.

[15] D. S. Ivanov and L. V. Zhigilei, "Effect of pressure relaxation on the mechanisms of short-pulse laser melting," Physical Review Letters, vol. 91, no. 10, Article ID 105701, 4 pages, 2003.

[16] S. I. Kudryashov, V. D. Zvorykin, A. A. Ionin, V. Mizeikis, S. Juodkazis, and H. Misawa, "Acoustic monitoring of microplasma formation and filamentation of tightly focused femtosecond laser pulses in silica glass," Applied Physics Letters, vol. 92, no. 10, Article ID 101916, 2008.

[17] C. Hu and J. R. Whinnery, "New thermooptical measurement method and a comparison with other methods," Applied Optics, vol. 12, no. 1, pp. 72-79, 1973.

[18] M. Sakakura and M. Terazima, "Oscillation of the refractive index at the focal region of a femtosecond laser pulse inside a glass," Optics Letters, vol. 29, no. 13, pp. 1548-1550, 2004.

[19] M. Sakakura and M. Terazima, "Initial temporal and spatial changes of the refractive index induced by focused femtosecond pulsed laser irradiation inside a glass," Physical Review B, vol. 71, no. 2, Article ID 024113, 12 pages, 2005.

[20] M. Sakakura, M. Terazima, Y. Shimotsuma, K. Miura, and K. Hirao, "Observation of pressure wave generated by focusing a femtosecond laser pulse inside a glass," Optics Express, vol. 15, no. 9, pp. 5674-5686, 2007.

[21] M. Sakakura, M. Terazima, Y. Shimotsuma, K. Miura, and K. Hirao, "Heating and rapid cooling of bulk glass after photoexcitation by a focused femtosecond laser pulse," Optics Express, vol. 15, no. 25, pp. 16800-16807, 2007.

[22] T. Hara, N. Hirota, and M. Terazima, "New application of the transient grating method to a photochemical reaction: the enthalpy, reaction volume change, and partial molar volume measurements," Journal of Physical Chemistry, vol. 100, no. 24, pp. 10194-10200, 1996. 
[23] M. Terazima, "Ultrafast transient Kerr lens in solution detected by the dual-beam thermal-lens method," Optics Letters, vol. 20, no. 1, pp. 25-27, 1995.

[24] M. Terazima, "Ultrafast rise of translational temperature after photoexcitation to electronic excited state in solution: transient lens study of $\mathrm{Ni}$ aqueous solution," Journal of Chemical Physics, vol. 105, no. 16, pp. 6587-6595, 1996.

[25] M. Pons, S. Nonell, I. Garcia-Moreno, A. Costela, and R. Sastre, "Time-resolved thermal lens study on the heat dissipation effects in solid polymeric matrices used as laser dyes," Applied Physics B, vol. 75, no. 6-7, pp. 687-694, 2002.

[26] M. Sakakura, S. Yamaguchi, N. Hirota, and M. Terazima, "Dynamics of structure and energy of horse carboxymyoglobin after photodissociation of carbon monoxide," Journal of the American Chemical Society, vol. 123, no. 18, pp. 42864294, 2001.

[27] J. F. Power, "Pulsed mode thermal lens effect detection in the near field via thermally induced probe beam spatial phase modulation: a theory," Applied Optics, vol. 29, no. 1, pp. 52 63, 1990.

[28] P. Martin, S. Guizard, Ph. Daguzan et al., "Subpicosecond study of carrier trapping dynamics in wide-band-gap crystals," Physical Review B, vol. 55, no. 9, pp. 5799-5810, 1997.

[29] M. Born and E. Wolf, Principle Optics, Cambridge University Press, Cambridge, UK, 7th edition, 1999.

[30] L. D. Landau and E. M. Lifshitz, Theory of Elasticity, Pergamon, Oxford, UK, 1986.

[31] I. Itzkan, D. Albagli, M. L. Dark, L. T. Perelman, C. von Rosenberg, and M. S. Feld, "The thermoelastic basis of short pulsed laser ablation of biological tissue," Proceedings of the National Academy of Sciences of the United States of America, vol. 92, no. 6, pp. 1960-1964, 1995.

[32] Data sheet from Matsunami Glass Ind.,Ltd.

[33] Z. Rosenberg, N. K. Bourne, and J. C. F. Millett, "Direct measurements of strain in shock-loaded glass specimens," Journal of Applied Physics, vol. 79, no. 8, pp. 3971-3974, 1996.

[34] A. Vogel, S. Busch, and U. Parlitz, "Shock wave emission and cavitation bubble generation by picosecond and nanosecond optical breakdown in water," Journal of the Acoustical Society of America, vol. 100, no. 1, pp. 148-165, 1996.

[35] E. N. Glezer, C. B. Schaffer, N. Nishimura, and E. Mazur, "Minimally disruptive laser-induced breakdown in water," Optics Letters, vol. 22, no. 23, pp. 1817-1819, 1997.

[36] G. Ghosh, "Model for the thermo-optic coefficients of some standard optical glasses," Journal of Non-Crystalline Solids, vol. 189, no. 1-2, pp. 191-196, 1995.

[37] Data sheets from Shinetsu.

[38] J. E. Shelby, "Density of vitreous silica," Journal of NonCrystalline Solids, vol. 349, no. 1-3, pp. 331-336, 2004.

[39] E. A. Vanina, M. A. Chibisova, and A. N. Chibisov, "Effect of $\gamma$-radiation on the optical properties of soda-lime-silicate glasses," Technical Physics Letters, vol. 33, no. 11, pp. 980-982, 2007.

[40] M. Sakakura, M. Shimizu, Y. Shimotsuma, K. Miura, and K. Hirao, "Temperature distribution and modification mechanism inside glass with heat accumulation during $250 \mathrm{kHz}$ irradiation of femtosecond laser pulses," Applied Physics Letters, vol. 93, no. 23, Article ID 231112, 2008.

[41] Data sheet from Corning Inc.

[42] G. S. Fulcher, "Analysis of recent measurements of the viscosity of glasses," Journal of the American Ceramic Society, vol. 8, no. 6, pp. 339-355, 1925.

[43] E. L. Cussler, Diffusion, Cambridge University Press, Cambridge, UK, 1984.
[44] C. A. Coulson and T. W. Dingle, "Structural crystallography and crystal chemistry," Acta Crystallographica Section B, vol. 24, no. 1, pp. 153-155, 1968. 